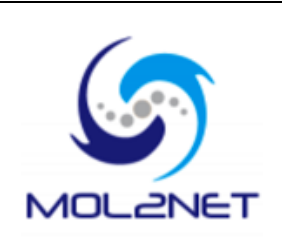

\title{
Building a New High-Selective Molecular Imprinted Polymer
}

\author{
Riccardo Concu *, Maria Natalia DiasSoeiro Cordeiro * \\ REQUIMTE, Department of Chemistry and Biochemistry, Faculty of Sciences, University of Porto, \\ Rua do Campo Alegre, 687, 4169-007 Porto, Portugal \\ * Correspondence: ric.concu@gmail.com, ncordeir@fc.up.pt
}

\section{Published: 8 December 2015}

\begin{abstract}
Molecular imprinted polymers (MIP) allows the preparation of tailored and high specific materials able to recognize a specific template. In this work, we simulated the affinity of a new high selective MIP able to specifically bind the isobutylphenylpropanoic acid (ibuprofen, template molecule). We have performed a series of molecular dynamics (MD) simulations of different mixtures in order to undercover the mechanisms occurring during the process of molecular imprinted polymers. The simulations were performed using the GROMACS 5.0 and the the OPLS-AA force field were used to parameterize and verifiy the studied molecules. A single system were simulated representing the pregelification state of the system. The radial distribution function (RDF) analysis and cluster analysis were used to evaluate the affinity of the template molecule, ibuprofen, for the gel backbone. Results confirm that the new material is high-selective and MD simulations are essential to study the molecular imprinting process because can give a deeper knowledge of the mechanism occurring during the imprinting process.
\end{abstract}

Keywords: Molecular imprinted polymers (MIP), molecular dynamics (MD), GROMACS, xerogel, polymers, isobutylphenylpropanoic acid.

\section{Introduction}

In recent years a new methodology have been developed to produce new and high selective polymers. Molecular imprintedpolymers (MIT) is a breaking through technology which is growing faster in these last years. For instance, the MITcan be used to prepare molecular imprinted polymers (MIP) that can be used to prepare synthetic receptors able to recognize and bind or release the template molecules, new HPLC

matrix for selective detection and/or separation of drugs.In this context, MIPmaterials are gaining day by day a most relevant role due to the growing demand for sensitive, accurate and simple methods and materials able to achieve this goal. In fact, MIPare widely used because they are able to recognize small chemicals or large biological molecules such as, proteins, DNA or RNA. In addition, MIP can be used to create 
sorbents for specific chiral chromatographic materials or specific sorbents for highperformance liquid chromatography-ultraviolet detection (HPLC-UV). The creation of new drug release materials is also an application of sol-gel MIP.

In this short communication, we present a Molecular Dynamics (MD) simulationof a new MIP, with a high selectivity for the isobutylphenylpropanoic acid (ibuprofen, template molecule). The radial distribution function (RDF) analysis has been used to evaluate the affinity between the MIP and the template molecule. This is a preliminar study to assess the affinity between the polymer and a template molecule which is a modification of a ORMOSIL we have recently published[1].

\section{Results and Discussion}

The RDF analysis was used to study the affinity between the template and the polymer. As referred in the materials and method section, the RDF was calculated using a specific atom instead of the center of the mass of the molecule. The atoms used for the template are the two oxygens of the carboxylic terminal, while for the polymer the hydrogen of the dehydroimidazolium; these atoms have been circle-marked in the Figure 1. In the Figure $\mathbf{2}$ we have reported the RDF analysis between $\mathrm{IBU}^{-}$(the template molecule) and a cationic dehydroimidazolium ORMOSIL $\left.\left(\mathrm{DHI}^{+}, \quad\left[\mathrm{Si}_{3} \mathrm{O}_{3}\left(\mathrm{CH}_{3}\right)_{4}(\mathrm{OH})\right)_{2}-\left(\mathrm{C}_{3} \mathrm{H}_{6}\right)\right]_{2}-\mathrm{C}_{3} \mathrm{H}_{5} \mathrm{~N}_{2}{ }^{+}\right)$. In the image is clear the affinity between the template and the polymer; in fact, there are two sharp and high peaks at a distance of $0.25 \mathrm{~nm}$ which clearly confirms the affinity. This peaks in fact correspond to the two oxygens of the template interacting with the hydrogen of the dihydroimidazolium of the ORMOSIL. In addition, in the same figure we have reported the RDF of the ORMOSIL with the counter ion, but in this case there is no relevant affinity. Thus, this result can confirms that is likely to happen a successfully imprinting effect.

Figure 1. IBU ${ }^{-}$and ORMOSIL structure

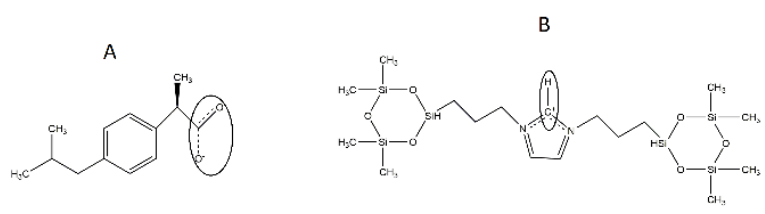

Figure 2. RDF analysis.

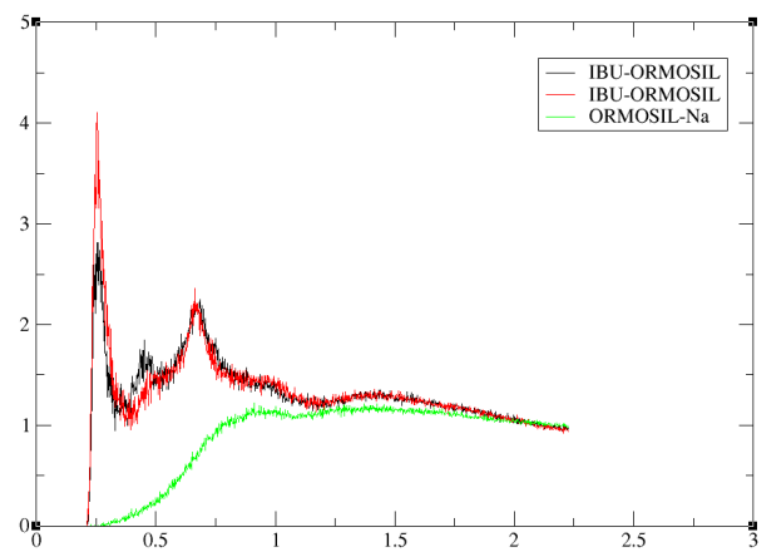

\section{Materials and Methods}

The MD simulations were performed with GROMACS 5.0.4.package applying the OPLSAA[2,3]. The system under study contained water, methanol, the anyonic form of Ibuprofen (the template, $\mathrm{IBU}^{-}$), the dual cyclic silicate trimer corresponding to a hydrolyzed and 
condensed species derived from the cationic dehydroimidazolium ORMOSIL (DHI $\left.\left.\left[\mathrm{Si}_{3} \mathrm{O}_{3}\left(\mathrm{CH}_{3}\right)_{4}(\mathrm{OH})\right)_{2}-\left(\mathrm{C}_{3} \mathrm{H}_{6}\right)\right]_{2}-\mathrm{C}_{3} \mathrm{H}_{5} \mathrm{~N}_{2}{ }^{+}\right)$, in the Figure 1 we have reported the ORMOSIL and the IBU ${ }^{-}$structures.The initial state of the system was obtained by inserting into the boxes the respective number of units at random positions using the packmol package[4]. The composition of the model is reported in the Table 1. After energy minimization using steepest-descent methods included in the GROMACS package, a temperature annealing was performed in the $N V T$ ensemble for $1 \mathrm{~ns}$, reaching a temperature of 600 $\mathrm{K}$, so as to ensure a proper mixing and gather three random independent initial configuration. Then the system were simulated for a total of $20 \mathrm{~ns}$ in the $N p T$ ensemble for data collection.Observable properties were sampled every 2 ps, from which total averages and standard deviations for each run were computed.The analysis consisted essentially in the calculation of radial distribution functions (RDF). The RDF between different types of molecules has been calculated as:
$g_{A B}(r)=\frac{\left\langle\rho_{B}(r)\right\rangle}{\left\langle\rho_{B}\right\rangle_{l o c}}$,

where $\left\langle\rho_{B}(r)>\right.$ refers to the average density of particle $\mathrm{B}$ at a distance $r$, around the particle $\mathrm{A}$, and $\left\langle\rho_{B}\right\rangle_{\text {loc }}$ refers to the density of the particle B averaged over all spheres around particles A with a maximum radius $\left(r_{\max }\right)$ which was half of the box length.

Table 1. Composition of the model

\begin{tabular}{cc} 
Molecule & Number \\
\hline Ibuprofen & 10 \\
Na & 20 \\
Water & 230 \\
Methanol & 1130 \\
Iodum & 20 \\
Ormosil & 10
\end{tabular}

\section{Conclusions}

This is only a preliminary work in order to assess the affinity between the template and the ORMOSIL molecule. Considering the reported results, we can affirm that a molecular imprinting process in this system is likely to happen. In addition, this work demonstrates that MD simulations could be useful to undercover atomistic basis of a imprinted process.

\section{Acknowledgments}

This work received financial support from the European Union (FEDER funds through COMPETE) and National Funds (FCT, Fundação para a Ciência e a Tecnologia) through projects Pest-C/EQB/LA0006/2013 (REQUIMTE) and Pest-C/QUI/UI0081/2013 (CIQ). The work also received financial support from the European Union (FEDER funds) under the framework of QREN through Project NORTE-07-0124-FEDER000067-NANOCHEMISTRY. RC acknowledges also FCT and the European Social Fund for financial support (Grant SFRH/BPD/80605/2011). To all financing sources the authors are greatly indebted. 


\section{References and Notes}

1. Concu, R.; Perez, M.; Cordeiro, M.N.; Azenha, M. Molecular dynamics simulations of complex mixtures aimed at the preparation of naproxen-imprinted xerogels. Journal of chemical information and modeling 2014, 54, 3330-3343.

2. Van Der Spoel, D.; Lindahl, E.; Hess, B.; Groenhof, G.; Mark, A.E.; Berendsen, H.J. Gromacs: Fast, flexible, and free. Journal of computational chemistry 2005, 26, 1701-1718.

3. Jorgensen, W.L.; Maxwell, D.S.; Tirado-Rives, J. Development and testing of the opls all-atom force field on conformational energetics and properties of organic liquids. Journal of the American Chemical Society 1996, 118, 11225-11236.

4. Martinez, L.; Andrade, R.; Birgin, E.G.; Martinez, J.M. Packmol: A package for building initial configurations for molecular dynamics simulations. Journal of computational chemistry 2009, $30,2157-2164$.

(C) 2015 by the authors; licensee MDPI, Basel, Switzerland. This article is an open access article distributed under the terms and conditions defined by MDPI AG, the publisher of the Sciforum.net platform. Sciforum papers authors the copyright to their scholarly works. Hence, by submitting a paper to this conference, you retain the copyright, but you grant MDPI AG the non-exclusive and unrevocable license right to publish this paper online on the Sciforum.net platform. This means you can easily submit your paper to any scientific journal at a later stage and transfer the copyright to its publisher (if required by that publisher). (http://sciforum.net/about). 